\title{
QUEST FOR GHZ STATES
}

\author{
M. ŻUKOWSKi ${ }^{a, b}$, A. ZEILINGER ${ }^{a}$, \\ M.A. HORNE ${ }^{c}$, AND H. WEINFURTER ${ }^{a}$ \\ ${ }^{a}$ Institut für Experimentalphysik, Universität Innsbruck, 6020 Innsbruck, Austria \\ ${ }^{b}$ Institute of Theoretical Physics and Astrophysics \\ Gdańsk Uniwersity, 80-952 Gdanisk, Poland \\ ${ }^{c}$ Stonehill College, North Easton, Massachusetts 02357, USA
}

\begin{abstract}
The premises of the Einstein-Podolsky-Rosen argument for their claim that quantum mechanics is an incomplete theory are inconsistent when applied to three-particle systems in entangled Greenberger-Horne-Zeilinger states. However, thus far there is no experimental confirmation for existence of such states. We propose a technique to obtain Greenberger-Horne-Zeilinger states which rests upon an observation that when a single particle from two independent entangled pairs is detected in a manner such that it is impossible to determine from which pair the single came, the remaining three particles become entangled.
\end{abstract}

PACS numbers: $03.65 . \mathrm{Bz}, 42.50 . \mathrm{Dv}, 89.70 .+\mathrm{c}$

\section{Introduction}

The premises of the Einstein-Podolsky-Rosen (EPR) argument [1] to show incompleteness of quantum mechanics are inconsistent when applied to maximally entangled states of at least three particles. Take a Greenberger-Horne-Zeilinger (GHZ) state of three particles which are on their way to three spatially separated observers (Fig. 1) [2]. Imagine that the relevant degrees of freedom for the local measurements are described by

$$
|\psi(3)\rangle=\frac{1}{\sqrt{2}}\left(|a\rangle|b\rangle|c\rangle+\left|a^{\prime}\right\rangle\left|b^{\prime}\right\rangle\left|c^{\prime}\right\rangle\right)
$$

where $\left\langle x \mid x^{\prime}\right\rangle=0(x=a, b, c$, and kets denoted by one letter pertain to one of the particles). The three observers $A, B$ and $C$ (spatially separated) measure the observables: $\widehat{A}\left(\phi_{A}\right), \widehat{B}\left(\phi_{B}\right), \widehat{C}\left(\phi_{C}\right)$, defined by

$$
\widehat{X}\left(\phi_{X}\right)=\left|+, \phi_{X}\right\rangle\left\langle+, \phi_{X}|-|-, \phi_{X}\right\rangle\left\langle-, \phi_{X}\right|
$$

and

$$
\left| \pm, \phi_{X}\right\rangle=\frac{1}{\sqrt{2}}\left( \pm \mathrm{i}\left|x^{\prime}\right\rangle+\exp \left(\mathrm{i} \phi_{X}\right)|x\rangle\right),
$$




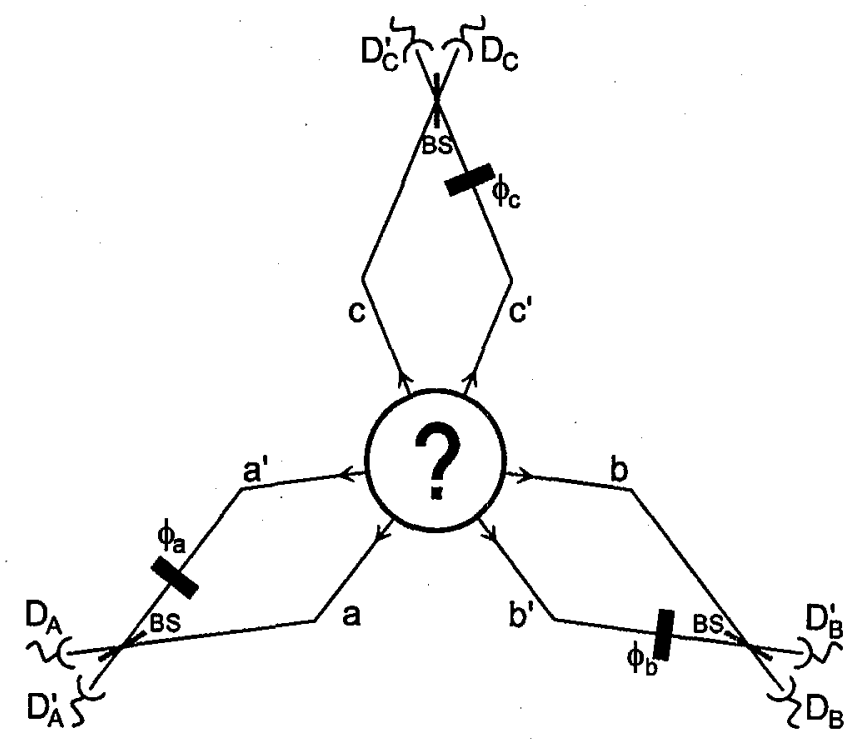

Fig. 1. Three-particle beam-entanglement GHZ interferometer [2]. Three particles in state (1) enter the arrangement. Adjustable phase shifters provide a change of the relative phase of the state components by $\phi_{A}+\phi_{B}+\phi_{C}$. Consequently the threefold coincident count rate in, say, detectors $D_{A}, D_{B}$, and $D_{C}$ will oscillate sinusoidally when the phase is varied. The detection events are spatially separated.

where $X=A, B, C$. The quantum prediction for the expectation value of the product of the three local observables is given by

$$
E\left(\phi_{A}, \phi_{B}, \phi_{C}\right)=\left\langle\psi\left|\widehat{A}\left(\phi_{A}\right) \widehat{B}\left(\phi_{B}\right) \widehat{C}\left(\phi_{C}\right)\right| \psi\right\rangle=\sin \left(\phi_{A}+\phi_{B}+\phi_{C}\right) .
$$

The EPR program, when applied to the above process, should be based first on the establishment of the elements of reality for the system. The EPR definition of an element of reality reads: if, without in any way disturbing a system, we can predict with certainty (i.e., with probability equal to unity) the values of a physical quantity, then there exist an element of physical reality corresponding to this physical quantity. The EPR argument continues now with the following steps. One establishes that quantum mechanics predicts for the studied system perfect correlations. This occurs in the present case when $\phi_{A}+\phi_{B}+\phi_{C}=\pi / 2+k \pi$ (that is, when $\sin (\pi / 2+k \pi)= \pm 1)$. E.g., for the settings $\phi_{A}=\pi / 2, \phi_{B}=0$ and $\phi_{C}=0$ whatever may be the results of local measurements of the observables, for say the particles belonging to the $i$-th triple (the ensemble of all such triples is represented by the quantum state $|\psi(3)\rangle)$, they have to satisfy

$$
A_{i}(\pi / 2) B_{i}(0) C_{i}(0)=1
$$

where $X_{i}(\phi)$ is the value of a local measurement of the observable $\widehat{X}(\phi)$ that would have been obtained for the $i$-th particle triple if the setting of the measuring device had been as indicated, i.e. $\phi$. Note that relativistic causality demands that $X_{i}(\phi)$ depends solely on the local parameter. Equation (5) clearly indicates that we can 
predict with certainty the result of measuring the observable pertaining to one of the particles (say $c$ ) by choosing to measure suitable observables for the other two. As the two first acts of measurement are spatially separated from the third particle, EPR argue that they cannot cause any real change in it. Hence the value $C_{i}(0)$ is an element of physical reality. had, e.g.

However, had the local apparatus settings been different one would have

$$
A_{i}(0) B_{i}(0) C_{i}(\pi / 2)=1 .
$$

We can repeat the argument, establishing now the value of the element of reality $C_{i}(\pi / 2)$. Hence two noncommuting observables $\widehat{C}(\pi / 2)$ and $\widehat{C}(0)$ are endowed with elements of reality (i.e. their values are predetermined). Since such statement is prohibited by quantum mechanics therefore it cannot be a complete theory!

However, for the three-particle state one can consider also two other similar situations and establish that quantum mechanical predictions imply that the elements of reality must satisfy

$$
A_{i}(0) B_{i}(0) C_{i}(\pi / 2)=1
$$

and

$$
A_{i}(\pi / 2) B_{i}(\pi / 2) C_{i}(\pi / 2)=-1
$$

The allowed values for the elements of reality are equal, by definition, to the eigenvalues of the respective observables, i.e. they are \pm 1 . If one multiples the left hand sides of Eqs. (5-8), and simply notices that all elements of reality appear in such a product twice, the result is, surprisingly, 1 , whereas, the product of right hand sides gives -1 . Thus the EPR program breaks down at the very outset, as their definition of the elements of reality (via the perfect correlations, and locality) is void. We have a "Bell theorem without inequalities" [2]. One can summarize the above story by yet another counterfactual statement (precisely on such type of statements the EPR argument is based on): had EPR known about GHZ states they might never have written their paper or, at least, they might have written it very differently.

Due to imperfections of laboratory devices, one cannot expect perfect correlations to occur. Thus, any test of local realism based on the GHZ correlations has to resort to some Bell-type inequalities. The simplest (ad hoc) algebraic form leading to a Bell-like inequality seems to be the one based upon the following algebraic identity:

$$
\begin{gathered}
A_{i}\left(\phi_{A}^{\prime}\right) B_{i}\left(\phi_{B}\right) C_{i}\left(\phi_{C}\right)+A_{i}\left(\phi_{A}\right) B_{i}\left(\phi_{B}\right) C_{i}\left(\phi_{C}^{\prime}\right)+A_{i}\left(\phi_{A}\right) B_{i}\left(\phi_{B}^{\prime}\right) C_{i}\left(\phi_{C}\right) \\
-A_{i}\left(\phi_{A}^{\prime}\right) B_{i}\left(\phi_{B}^{\prime}\right) C_{i}\left(\phi_{C}^{\prime}\right)= \pm 2,
\end{gathered}
$$

which must be always satisfied if $X_{i}\left(\phi_{X}\right)= \pm 1$. Thus the average values for local realistic [3] predictions for the products of the local results should fulfill

$$
\begin{aligned}
-2 \leq & E\left(\phi_{A}^{\prime}, \phi_{B}, \phi_{C}\right)+E\left(\phi_{A}, \dot{\phi}_{B}, \phi_{C}^{\prime}\right)+E\left(\phi_{A}, \phi_{B}^{\prime}, \phi_{C}\right) \\
& -E\left(\phi_{A}^{\prime}, \phi_{B}^{\prime}, \phi_{C}^{\prime}\right) \leq 2 .
\end{aligned}
$$

The maximal violation of this inequality, by a factor of 2 , is obtained if we put into it the quantum prediction with the previously discussed settings: $\phi_{X}=0, \phi_{X}^{\prime}=\pi / 2$. 
In a real experiment one cannot expect the three-particle interference fringes to be of $100 \%$ visibility $(V)$ and therefore one expects the correlation function to be $V \sin \left(\phi_{A}+\phi_{B}+\phi_{C}\right)$ [4]. Therefore, the critical value of the visibility to violate the Bell inequality is $V_{\text {crit }}=50 \%$ (i.e. much lower than the threshold for the two-particle Bell-type experiment).

\section{Experiment}

While it would be interesting to experimentally exhibit the correlations present in a three-particle entanglement, no experimentally tested procedure exists for preparing the necessary state. The natural source of three-particle entanglements, three-photon positonium annihilation, is a rare event, and the polarizations of the $\gamma$ rays are difficult to measure. Also one could think of a higher order spontaneous down-conversion process involving cubic nonlinearity in crystal's polarizability. However, both processes share (almost) complete unpredictability of the directions of emission (this makes the count rates very low). Since 1989, many other sources were proposed but thus far no experiment has been performed [5].

We wish to propose a possibly realizable, relatively simple procedure for generating three-particle entanglement out of only two pairs of entangled photons [6]. One can generate these states by interfering photon pairs produced by two independent spontaneous parametric frequency down-conversion (PDC) sources. The product of two two-particle states can be projected, by a measurement upon one of the four particles, in such a way that the resulting collapse leads to a three-particle GHZ-state. As the PDC sources are endowed with high angular correlation of the emissions, this feature is also present in our compound source (what, despite relatively low probability of simultaneous emissions from two independent sources, should contribute to tolerable count rates).

Yurke and Stoler [7] were first to suggest that interfering particles from independent sources may give rise to non-classical Bell-EPR correlations. Interference between particles produced by independent sources is observable only if the origin of the particles cannot be inferred anymore [8]. This necessitates that either the coincidence detection or the generation of the particles is done with a time resolution shorter than their coherence times [9]. Here we shall employ the second alternative.

Consider the arrangement of Fig. 2. Two independent PDC sources each emit a pair of particles in a beam entangled state (type-I phase matching) [10] and, by chance, these emissions are nearly simultaneous. Suppose for example that the states of the pairs are

$$
\frac{1}{\sqrt{2}}\left(|a\rangle|d\rangle+\left|a^{\prime}\right\rangle\left|c^{\prime}\right\rangle\right)
$$

from source $A$, and

$$
\frac{1}{\sqrt{2}}\left(\left|d^{\prime}\right\rangle\left|b^{\prime}\right\rangle+|c\rangle|b\rangle\right)
$$

from source $B$ (the letters represent beams taken by the photons in Fig. 2, all beams have the same polarization) [10]. The beams $d$ and $d^{\prime}$ are mixed by a $50-50$ beamsplitter, behind which are two detectors $D_{\mathrm{T}}$ (trigger) and $D_{\mathrm{T}}^{\prime}$. 


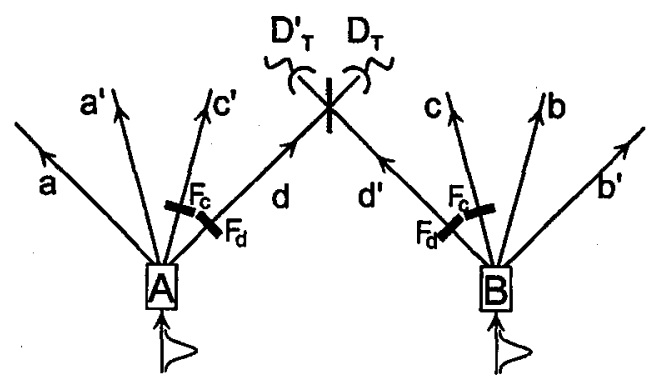

Fig. 2. A three-particle beam-entanglement source. Short pulses of duration $\Delta T$ stimulate two independent two-particle sources, $A$ and $B$, to each emit a pair of beam-entangled particles. The state of the pair from $A[B]$ is given by Eq. (11) [(12)]. Suppose that the trigger detector, $D_{\mathrm{T}}$, registers a single particle and the other three particles are eventually found to have been in beams $a$ or $a^{\prime}, b$ or $b^{\prime}$, and in $c$ or $c^{\prime}$, respectively. If the trigger particle came from $A$ via transmission at the beam splitter, $B S$, its sibling must be in beam $a$ and the pair from $B$ must be in beams $b$ and $c$. If the trigger particle came from $B$ via reflection at $B S$, its sibling must be in beam $b^{\prime}$ and the pair from $A$ must be in beams $a^{\prime}$ and $c^{\prime}$. Narrow filters, $F_{c}$ and $F_{d}$, of widths much narrower than the pulse spectrum make the source of the trigger particle essentially unknowable (see text). Consequently, the state of the other three particles is the entanglement of Eq. (13).

Suppose that one and only one of these four particles is detected by $D_{\mathrm{T}}$, no particle is detected at $D_{\mathrm{T}}^{\prime}$, and the other six beams illuminate the three-particle interferometer [2] of Fig. 1. Because of the beamsplitter, the trigger particle could have come from either source $A$ or $B$. If it came from $A$, its companion must be in beam $a$, and the pair from $B$ must be in beams $b$ and $c$. Thus, the state of the triple of remaining particles is $|a\rangle|b\rangle|c\rangle$. If on the other hand the trigger particle came from source $B$, its companion must be in beam $b^{\prime}$ and the pair from $A$ must be in beams $a^{\prime}$ and $c^{\prime}$. Thus, if the trigger particle came from $B$, the state of the remaining triple is $\left|a^{\prime}\right\rangle\left|b^{\prime}\right\rangle\left|c^{\prime}\right\rangle$.

Now, if the procedure of emission and selection of the four photons is such that one cannot ever know, not even in principle, which source produced the trigger event, then the other photons, as they enter the interferometer of Fig. 1, will be in a superposition of the two three-particle states mentioned above, i.e. in the GHZ state

$$
\frac{1}{\sqrt{2}}\left(|a\rangle|b\rangle|c\rangle+\mathrm{e}^{\mathrm{i} \phi}\left|a^{\prime}\right\rangle\left|b^{\prime}\right\rangle\left|c^{\prime}\right\rangle\right)
$$

where the relative phase $\phi$ depends on the positions of various elements of the full setup.

For the coherent superposition of state (13) to form one must erase all ways by which one might in principle identify true pairs. We assume that the two sources are pumped by one pulsed laser and emitting down converted radiation of a degenerate frequency. All three remaining photons (after the trigger event) are fed into three spatially separated interferometers (see, Fig. 1). The detection 
station $B$ observes the radiation coming from beams $b$ and $b^{\prime}$, station $A$ from beams $a$ and $a^{\prime}$, whereas the station $C$ collects the radiation from $c$ and $c^{\prime}$. In all beams we have filters of the same central frequency (half of the pump frequency). However, the widths of the filters may differ.

Suppose that the three photons and the trigger one are detected coincidently (within a few nanosecond window), one in each detection stations $A, B$ and $C$, and the fourth at $D_{\mathrm{T}}$. Clearly the photons at the first two stations came from the crystals bearing the same name as the station. But the origins of the photon at $D_{\mathrm{T}}$ and $C$ are unclear. In such a case one could, in principle, determine that photon detected at $D_{\mathrm{T}}$ came from crystal $A(B)$ by noting the near simultaneity of the detection of photon $D_{\mathrm{T}}$ and one of the photons at $B$ or $A$ (this is due to the property of the PDC radiation: the detection times of a pair are extremely well correlated). To ensure that the source of photons is unknowable, we propose that the two crystals should be pumped by pulses of durations around $\tau \approx 100 \mathrm{fsec}$, and that the trigger should be detected behind a narrow filter whose inverse of the bandwidth (coherence time) exceeds $\tau$. If one of the members of a PDC pair passes such a filter the temporal correlation with its companion spreads to around $\tau$. Thereby placing suitable filters prevents identification of the trigger photon and its partner by comparison of their arrival times. However, if the detections at $C$ and either $A$ or $B$ are strictly time correlated one still concludes that the photons came from one crystal. One can again remedy this by putting filters of coherence time exceeding $\tau$ in beams $c$ and $c^{\prime}$. Now, the which-way information is completely erased. Please note that due to the pulsed nature of the pump (i.e. its non-monochromaticity) the complementary feature which could in principle betray the origin of particles at $D_{\mathrm{T}}$ and $C$, namely the frequency correlation, is more fuzzy than the frequency resolution imposed by the filters. We can expect high visibility of three-particle fringes.

With a more quantitative description we now shall estimate the visibility of the three-particle fringes. The following assumptions will be made: (i) the probability of a multiple emission from a single PDC is low; (ii) the pulse is not too short, i.e., the nonmonochromaticity of the pulse will not blur too much the strong angular correlation of the emissions (due to the effective energy and momentum conservation within the crystal). Thus, the photons can be described as emitted in specified, very well-defined directions.

The state of the photon pair emerging from the source $A$ (plus the filter system) can therefore be approximated by

$$
\begin{aligned}
\left|\Psi^{A}\right\rangle & =\left|\psi_{a d}\right\rangle+\left|\psi_{a^{\prime} c^{\prime}}\right\rangle=\int \mathrm{d} \omega_{1} \mathrm{~d} \omega_{2} \mathrm{~d} \omega_{0} \Delta\left(\omega_{0}-\omega_{1}-\omega_{2}\right) g\left(\omega_{0}\right) \\
& \times\left(f_{a}\left(\omega_{1}\right) f_{d}\left(\omega_{2}\right)\left|\omega_{1} ; a\right\rangle\left|\omega_{2} ; d\right\rangle+f_{a^{\prime}}\left(\omega_{1}\right) f_{c^{\prime}}\left(\omega_{2}\right)\left|\omega_{1} ; a^{\prime}\right\rangle\left|\omega_{2} ; c^{\prime}\right\rangle\right),
\end{aligned}
$$

where, e.g., the ket $|\omega, e\rangle$ describes a single photon of frequency $\omega$ in the beam $e$, the function $g$ represents the spectral content of the pulse, and $f_{e}$ is the transmission function of the filter in the beam $e$. The function $\Delta$ is sharply peaked at the origin and describes the phase-matching condition. One can approximate it by the Dirac delta. Similar structure has the ket describing the pair of photons coming from the other source, $\left|\psi_{B}\right\rangle$. 
If one introduces objects [9] like, e.g. $|t ; b\rangle=(1 / \sqrt{2 \pi}) \int \mathrm{d} \omega \mathrm{e}^{\mathrm{i} \omega t}|\omega ; b\rangle$, the amplitude, e.g., to detect a photon at time $t_{x^{\prime}}$ by a detector monitoring the beam $x^{\prime}$ and another one at time $t_{y^{\prime}}$ by a counter in the beam $y^{\prime}$, provided the initial photon state was, say, $\left|\psi_{x y}\right\rangle$, can be written as $A_{x y}\left(t_{x^{\prime}}, t_{y^{\prime}}\right)=\left(\left\langle t_{x^{\prime}} ; x^{\prime}\left|\left\langle t_{y^{\prime}} ; y^{\prime}\right|\right) \mid \psi_{x y}\right\rangle\right.$. The elementary amplitudes of the interferometric process have now a simple, intuitively appealing, form $\left.A_{x y}\left(t_{x}, t_{y}\right)=(1 / \sqrt{2 \pi}) \int \mathrm{d} t G(t) F_{x}\left(t_{x}-t\right) F_{b}\left(t_{y}-t\right)\right)$, where the functions denoted by capitals are the Fourier transforms: $F(t)=(1 / \sqrt{2 \pi}) \times$ $\int \mathrm{d} \omega \mathrm{e}^{\mathrm{i} \omega t} f(\omega)$.

The amplitude of the three-photon interference at, say, detectors $D_{A}, D_{C}$ and $D_{B}$ (detector $D_{X}$ registers photons in state $\left|+, \phi_{X}\right\rangle$ ), at times $t_{A}, t_{B}$ and $t_{C}$, under the condition that the trigger photon fired at $t_{\mathrm{T}}$, is proportional to

$$
A_{a d}\left(t_{A}, t_{\mathrm{T}}\right) A_{c b}\left(t_{C}, t_{B}\right)+\mathrm{e}^{\mathrm{i} \phi} \mathrm{e}^{\mathrm{i}\left(\phi_{a}+\phi+b \phi_{c}\right)} A_{b^{\prime} d^{\prime}}\left(t_{B}, t_{\mathrm{T}}\right) A_{a^{\prime} c^{\prime}}\left(t_{A}, t_{C}\right),
$$

where $\phi_{i}, i=a, b, c$ is the local phase shift in the given beam. To get the overall probability of the process one has to integrate the square of the modulus of the amplitude over the detection times (the time resolution of the detectors is of the order of nanoseconds, which is much longer than the coherence times, thus due to our earlier assumptions the integration over time can be extended to infinity).

Now, if one assumes that the filters in beams leading to a single detector station are identical, that the functions have the following structure: $F_{f}(t)=$ $\mathrm{e}^{-\mathrm{i} \frac{1}{2} \omega_{\mathrm{p}} t}|F(t)|, G(t)=\mathrm{e}^{-\omega_{\mathrm{p}} t}|G(t)|$, where $\omega_{\mathrm{p}}$ is the central frequency of the pulse, then the maximal visibility of the three-particle interference can be written as

$$
V(3)=\frac{\int \mathrm{d}^{4} t\left|A_{a d}\left(t_{A}, t_{\mathrm{T}}\right) A_{b c}\left(t_{B}, t_{C}\right) A_{b^{\prime} d^{\prime}}\left(t_{B}, t_{\mathrm{T}}\right) A_{a^{\prime} c^{\prime}}\left(t_{A}, t_{C}\right)\right|}{\int \mathrm{d}^{4} t\left|A_{a d}\left(t_{A}, t_{\mathrm{T}}\right) A_{b c}\left(t_{B}, t_{C}\right)\right|^{2}},
$$

where $\mathrm{d}^{4} t=\mathrm{d} t_{A} \mathrm{~d} t_{B} \mathrm{~d} t_{C} \mathrm{~d} t_{T}$.

If one specifies, for simplicity, all the functions as Gaussians, $\exp \left[-\frac{(\omega-\Omega)}{2 \sigma^{2}}\right]$, where $\Omega$ is the mid frequency and $\sigma$ the width, the formula for the visibility reads

$$
V(3)=\left(\frac{2 \sigma_{\mathrm{p}}^{2}}{2 \sigma_{\mathrm{p}}^{2}+\sigma_{A}^{2} \sigma_{C}^{2} /\left(\sigma_{\mathrm{p}}^{2}+\sigma_{A}^{2}+\sigma_{C}^{2}\right)+\sigma_{A}^{2} \sigma_{T}^{2} /\left(\sigma_{\mathrm{p}}^{2}+\sigma_{A}^{2}+\sigma_{T}^{2}\right)}\right)^{1 / 2},
$$

where $\sigma_{\mathrm{p}}$ is the spectral width of the pulse, $\sigma_{f}, f=A, B, C$, is the width of the filter, and we assume that $\sigma_{A}=\sigma_{B}$. If one removes the filters $A$ and $B$, the formula simplifies to

$$
V(3)=\left(\frac{2 \sigma_{\mathrm{p}}^{2}}{2 \sigma_{\mathrm{p}}^{2}+\sigma_{C}^{2}+\sigma_{T}^{2}}\right)^{\frac{1}{2}}
$$

Therefore, narrow filters in paths $a, a^{\prime}$ and $b, b^{\prime}$ are not necessary to obtain high visibility. The other filters should be always sufficiently narrow.

\section{Prospects}

Currently realizable values of $\sigma_{f} \approx 1 \mathrm{~nm}$ for filter widths and $\sigma_{\mathrm{p}} \approx 5 \mathrm{~nm}$ for pulse spectral width yield $V(3) \approx 97 \%$. This is well above the required $50 \%$, and therefore there is much room before the imperfections of the actual equipment force down the visibility below this crucial figure. It is worthwhile to add that 
our current set-up for pulsed two-source down conversion (producing polarization entanglements [11]) gives about $10^{-2} \mathrm{~s}^{-1}$ fourfold coincidences, but with wider filters.

The principal aim of the experiment will be to show the existence of $\mathrm{GHZ}$ states. However, one also may view it as yet another attempt for a test against local realism. At the present moment plenty of "loophopes" seem to be present in our proposal if one wishes to view it in this way. However, if one attempts to interpret experimental observations in a local realistic way, one must notice that while the filters select photons, one could in principle detect all of them by using a suitably sophisticated dispersive optical element. The other complications are less important. For example, the trigger detector may fire if (a) only one down conversion occurred, (b) two down conversions occurred in one crystal. Case (a) can be rejected, two of the detector stations will show no counts. Case (b) can also be rejected, station $D_{A}$ or $D_{B}$ will exhibit no counts.

The laboratory realization of three-particle entanglement will open the door to many novel quantum phenomena and applications. These may include, not only the demonstration of GHZ correlations, but also: (a) generalization of two-particle phenomena (e.g. illumination of a tritter [12] with three entangled particles), (b) demonstration of entangled entanglement [13], (c) multiparticle quantum communication schemes (see, e.g. [14]).

One can for example link the ideas (b) and (c) and notice that the use of GHZ states (1) makes possible generation of a cryptographic key in such a way that it can be controlled by a third operator who decides whether to activate the key or not. Imagine that Alice, Bob and Cecil observe GHZ correlations. The protocol of key establishment between Alice and Bob is exactly the same as the one proposed by Ekert [15] for standard two-particle EPR-Bell correlations. However, depending on the measurement result at the station of Cecil, Alice and Bob share different two photon entangled states. Say that Cecil's observable is given by (3) with $\phi_{C}=\pi / 2$. Then depending on the local result that he obtains the state shared by Alice and Bob is either $|\psi(2+)\rangle=(1 / \sqrt{2})\left(|a\rangle|b\rangle+\left|a^{\prime}\right\rangle\left|b^{\prime}\right\rangle\right)$ or $|\psi(2-)\rangle=$ $(1 / \sqrt{2})\left(|a\rangle|b\rangle-\left|a^{\prime}\right\rangle\left|b^{\prime}\right\rangle\right)$, and therefore he holds the key to their key. Without the information on his results all that Alice and Bob share is just useless. However, once Cecil has communicated his results to Alice and Bob, the key they now can establish is also perfectly secret to him. The Third Man can control the key distribution but not the secret communication once he allows it!

This work was supported by the Austrian Fonds zur Förderung der wissenschaftl. Forschung (S6502). M. Zukowski acknowledges partial support of the University of Gdańsk research grant No. BW-5400-5-0306-7, and of the 1996/97 Austrian-Polish scientific-technological collaboration program No. 22 (Quantum communication and quantum information). H.W. is supported by the APART-fellowship of the Austrian Academy of Science. 


\section{References}

[1] A. Einstein, B. Podolsky, N. Rosen, Phys. Rev. 47, 777 (1935).

[2] D.M. Greenberger, M. Horne, A. Zeilinger, in: Bell's Theorem, Quantum Theory, and Conceptions of the Universe, Ed. M. Kafados, Kluwer, Dordrecht 1989; D.M. Greenberger, M.A. Horne, A. Shimony, A. Zeilinger, Am. J. Phys. 58, 1131 (1990); N.D. Mermin, Phys. Today, June 1990, p. 9.

[3] J.S. Bell, Physics 1, 195 (1965).

[4] M. Żukowski, Phys. Lett. A 177, 290 (1993).

[5] For example, K. Wódkiewicz, L.-W. Wang, J.H. Eberly, Phys. Rev. A 47, 3280 (1993); J.I. Cirac, P. Zoller, Phys. Rev. A 50, R2799 (1994); S. Haroche, Ann. N. Y. Acad. Sci. 755, 73 (1995); T. Sleator, H. Weinfurter, Ann. N. Y. Acad. Sci. 755, 646 (1995); F. Laloë, Current Science 68, 1026 (1995); C.C. Gerry, Phys. Rev. A 53, 2857 (1996); T. Pfau, C. Kurtsiefer, J. Mlynek, Quantum Semiclass. Opt. 8, 665 (1996).

[6] A. Zeilinger, in: 15 th Intern. Conf. Atomic Physics (ICAP), Ed. J. Walraven, Amsterdam 1996; A. Zeilinger, M.A. Horne, H. Weinfurter, M. Żukowski, Phys. Rev. Lett. 78, 3031 (1997).

[7] B. Yurke, D. Stoler, Phys. Rev. Lett. 68, 1251 (1992); Phys. Rev. A 46, 2229 (1992).

[8] M. Żukowski, A. Zeilinger, M.A. Horne, A. Ekert, Phys. Rev. Lett. 71, 4287 (1993).

[9] M. Żukowski, A. Zeilinger, H. Weinfurter, Ann. N. Y. Acad. Science 755, 91 (1995).

[10] M.A. Horne, A. Shimony, A. Zeilinger, Phys. Rev. Lett. 62, 2209 (1989); J.G. Rarity, P.R. Tapster, Phys. Rev. Lett. 64, 2495 (1990).

[11] P.G. Kwiat, K. Mattle, H. Weinfurter, A. Zeilinger, Phys. Rev. Lett. 75, 4337 (1995).

[12] A. Zeilinger, M. Żukowski, M.A. Horne, H.J. Bernstein, D.M. Greenberger, in: Quantum Interferometry, Eds. F. DeMartini, A. Zeilinger, World Scientific, Singapore 1994, p. 159; M. Żukowski, A. Zeilinger, M.A. Horne, Phys. Rev. A 55, 2564 (1997).

[13] G. Krenn, A. Zeilinger, Phys. Rev. A 54, 1793 (1996).

[14] C.H. Bennett, Phys. Today, Oct. 1995, p. 24.

[15] A.K. Ekert, Phys. Rev. Lett 67, 661 (1991). 